\title{
Neuroprotective effect of Estrogen Receptor a against neuroinflammation induced by hypoxia/ischemia via SIRT1-dependent AMPK pathway
}

\section{Z Z ( $D$ zhongze1012@163.com)}

Department of Cardiology,Jiande Branch,Second Affiliated Hospital,Zhejiang University School of Medicine

\section{He WQ}

Hubei province Key Laboratory of Occupational Hazard Identification and Control,Department of pharmacology,Medical college of Wuhan University of Science and Technology

\section{Cao XL}

Hubei Province Key Laboratory of Occupational Hazard Identification and Control,Departmnet of pharmacology,Medical college of Wuhan University of Science and Technology

\section{Tang B}

Department of Cardiology,Jiande Branch ,Second Affiliated Hospital,Zhejianng University School of Medicine

\section{Fan QY}

Department of Cardiology,Jiande Branch,Second Affiliated Hospital,Zhejiang University School of Medicine

\section{Zhao MH}

Hubei Province Key Laboratory of Occupational Hazard Indentification and Control,Department of pharmacology,Medical college of Wuhan University of Science and Technology

\section{Chen YJ}

Hubei Province Key Laboratory of Occupational Hazard Indentification and Control,Department of pharmacology,Medical of college of Science and Technology

\section{Research article}

Keywords: Cerebral Ischemia; NLPR3 inflammasome; Estrogen Receptor a; SIRT1; AMPK pathway

Posted Date: September 17th, 2019

DOI: https://doi.org/10.21203/rs.2.14472/v1 
License: (c) (i) This work is licensed under a Creative Commons Attribution 4.0 International License. Read Full License 


\section{Abstract}

Background: Stroke-related damage in rats is protected against by estrogen which also has an anticerebral ischemia role mostly conducted via its association with estrogen receptor (ER) a .However,processes governing ER a-mediated neuroprotection are poorly comprehended. This study sought to establish whether ERa's control of neuroinflammation caused by NLRP3 inflammasome activation emanating from SIRT1-AMPK signaling allowed ERa's improvement of hypoxia/ischemic damage.

Methods: The intraperitoneal administration of estrogen was performed to ovariectomized (bilaterally) (OVA) SD rats prior to middle cerebral artery occlusion (MCAO). The strong rise in NLRP3 inflammasome activation including caspase-1, ASC, IL-1 $\beta$ and IL-18 occurred following OVA and were specifically decreased following estrogen treatment. Moreover, the expression of Silent Information Regulator 1 (SIRT1) and ERa were reversed. The association between ERa-led inhibition of the NLRP3 inflammasome in conditions of hydrogen peroxide ( $\mathrm{H} 2 \mathrm{O} 2$ ) and SIRT1-AMPK signaling were also examined.

Results: Findings confirmed the prevention of NLRP3 inflammasome activation instigated by $\mathrm{H} 2 \mathrm{O} 2$ and the in vitro production of IL-1 1 , IL-18 together with the enhancement of this impact by SIRT1. Additionally, ERa's neuroprotective impact was prevented by inhibiting of AMPK. The synergistic impact of SIRT1 on ERa increased AMPK activation; however, SIRT1 knockout eliminated this.

Conclusions: The findings indicate that inhibition of the NLRP3 inflammasome by ERa results in neuroprotection against hypoxia/ischemic injury and that ERa's neuroprotection can be highly improved by the SIRT1-dependent AMPK pathway.

\section{Background}

High mortality and disability rates are associated with ischemic cerebral/reperfusion ${ }^{[1,2]}$, with complex processes governing the pathological mechanisms of ischemic cerebral/reperfusion damage ${ }^{[3-5]}$, including neurotoxin, neuroinflammation, oxidative stress and neuronal apoptosis. Till today the primary role of the neuroinflammatory reaction in cortical ischemic-reperfusion damage has been noted ${ }^{[6,7]}$. The CNS highly expresses NLRP3 (NOD-like receptor family, pyrin domain-containing 3); a complex of NLRP3 scaffold, caspase -1 and the ASC (PYCARD) adaptor ${ }^{[8,9]}$. Caspase- 1 activation results from NLRP3 inflammasome activation leading to the production of the pro-inflammatory cytokines interleukin-1 $\beta$ (IL$1 \beta)$ and interleukin-18 (IL-18) and initiation of prompt cell death ${ }^{[10]}$. Numerous studies linked cerebral ischemic-reperfusion development with the NLRP3 inflammasome ${ }^{[11,12]}$. Thus, possible therapeutic targets are found in the prevention of the neuronal inflammatory response.

The NAD+-dependent class III histone deacetylase SIRT1 (Silent information regulator 1 ) has a significant part in numerous biological functions such as oxidative stress, inflammation, metabolism and aging ${ }^{[13]}$. Recent data has indicated the inhibition of NFK Band p53-induced neuronal apoptosis and inflammatory 
responses by SIRT1 indicating SIRT1's key role in neuroprotection ${ }^{[14-16]}$. Furthermore, the prevention of NLRP3 activation and its anti-inflammatory impact is also mediated by SIRT1 ${ }^{[17-18]}$. Therefore, much notice has been given to the possibility of using the NLRP3 inflammasome-mediated by SIRT1 signaling pathway as a therapeutic target concerning cerebral ischemia.

The typical genomic reactions in target tissues born of estrogen release are mediated by two receptors of the steroid hormone receptor superfamily; namely estrogen receptor (ER) $a$ and $\beta^{[19]}$. Previous research indicated the need for ER $\beta$ in cortical neuronal migration during development but it was absent in decreasing ischemia-mediated injury [20-22]. Furthermore, these studies indicated ERa's facilitating of estrogen's neuroprotective impacts in the cerebral cortex in animals both old and young ${ }^{[23]}$, thus indicating ERa's principal role in protecting against cerebral ischemia-reperfusion injury. Interestingly, earlier research alluded to the inhibitory effect of estrogen on the expression of the NLRP3 inflammasome gene in the cerebral cortex following cerebral ischemia ${ }^{[24]}$, thereby proposing estrogen's regulation of inflammasome activation. Nevertheless, evidence is still required for estrogen-directed ERa expression suppression of the NLRP3 inflammasome via regulation of SIRT1 following cerebral ischemia.

This research aims to examine ERa processes and it's neuroprotective impact on neuroinflammation instigated by cerebral ischemia initiated by the SIRT1-dependent pathway.

\section{Methods}

\section{Reagents}

PC12 cells were obtained from the Cell bank of the Chinese Academy of sciences.

Genechem company (Shanghai, China) constructed all plasmids including wild type ERa, wild-type SIRT1 and SIRT1 siRNA. NLRP3 antibody was purchased from Adipogen (San Diego, USA). IL- $1 \beta$, IL- 18 , $\beta$-actin and Caspase-1 p10 antibodies were purchased from Santa Cruz Biotechnology (Dallas, USA). Fu GENE HD, FCM Assay System Kit was obtained from Roche (Diagnostics, Indianapolis).

\section{Animals}

The Laboratory Animal Center of Hubei Province supplied the female Sprague-Dawley (SD) rats with weights ranging between 280 to $320 \mathrm{~g}$. Rats were kept in pathogen-free environments and separated into sham, M\&OVA, MCAO and $M \& E_{2}$ groups. $10 \%$ chloral hydrate anesthesia was used prior to bilateral ovariectomization of the M\&OVA group by insertion of nylon monofilament into the anterior cerebral artery. Group prior to MCAO, M\&OVA groups: 7 days before OVA, the rats were administered estrogen $(0.5 \mathrm{mg} / \mathrm{kg})$ intraperitoneally before MCAO. Numbers of animals utilized and animal suffering was kept to a minimum. The protocol was approved by the Institutional Animal Care and Use Committee and the Medical ethics committee of wuhan university of science and technology (No:201961). 


\section{Middle cerebral artery occlusion/reperfusion model (MCAO)}

$350 \mathrm{mg} / \mathrm{kg}$ i.p. of $10 \%$ chloral hydrate was used to anesthetize rates by insertion of nylon monofilament (0.25-0.28mm diameter) into the anterior cerebral artery via the external carotid artery for $2 \mathrm{~h}$ prior to recovering perfusion. Focal cerebral ischemia was induced by occlusion of the right MCAO with a nylon monofilament (diameter criteria,0.25-0.28mm) with a heparin-coated tip (Huang et al., 1994). This coated filament was introduced into the internal carotidartery through the external carotid artery, up to the origin of the anterior cerebral artery, and occluded the MCA andanterior cerebral artery for $2 \mathrm{~h}$. Rats with a neurological score $<3$ were removed from the study. All rats were euthanized at ischemic $2 \mathrm{~h}$-reperfusion 24h. After the MCAO building, once rats lack the alertness of physical or mental that result from the symptoms of unconscious or bleeding, rats would be euthanasia by an overdose of pentobarbital (120 $\mathrm{mg} / \mathrm{kg}$ intraperitoneal injection) to produce unconsciousness and death ${ }^{[25,26]}$. The humane method we chosen is to induce a rapid, painless, and distress-free death.

\section{Infarct volume evaluation}

Coronal sectioning was performed on brains followed by TTC (Sigma, USA) staining for quantification by incubation in $2 \%$ TTC solution at $37^{\circ} \mathrm{C}$ for $20 \mathrm{~min}$ prior to paraformaldehyde (4\%) fixation. Infarct volume was calculated by totaling infarct area per section and multiplying it through the distance between section as indicated by image analysis software (Version 1.61, NIH image).

\section{Cell culture and Treatment}

PC12 cells were cultured in Ham's F12K Medium supplemented with FBS (2.5\%), horse serum (15\%), penicillin and streptomycin $(100 \mu \mathrm{g} / \mathrm{ml})$ and incubated in a humidified atmosphere containing $5 \% \mathrm{CO}_{2}$ at $37^{\circ} \mathrm{C}$. Wild-type ERa, SIRT1 or SIRT1 siRNA plasmids were used to transfect PC12 cells following their seeding and coating with poly-L-Lysine. Transfection was performed in Ham's F12K-Medium with DNA $(0.5 \mu \mathrm{g})$ and Fu GENE $(2.5 \mu \mathrm{l})$ prior to adding culture medium consisting of $400 \mu \mathrm{M}$ hydrogen peroxide.

\section{HE stains}

Brain pieces were fixed in Bouin's solution following washing in physiological saline. They were then dehydrated using increasing ethanol concentrations, paraffin embedded and cut to $2 \mathrm{~mm}$. Brain sections were hematoxylin-eosin (HE) stained and examined under the light microscope.

\section{Flow cytometric}

PC12 cells' viability was examined using flow cytometry (FCM) following PI and Annexin V-FITC staining. 


\section{Detection of $\mathrm{LDH}$ release}

The LDH-release kit (Nanjing Jincheng Bioengineering Institute) was used to quantify LDH as per manufacturer's instructions.

\section{Western blot analysis}

A cell pellet containing $50 \mu \mathrm{g}$ cells or cerebral cortex was collected and electrophoresed in sodium dodecyl sulfate-polyacrylamide gel (15\%) prior to membrane probing with antibodies (NLRP3, caspase-1 p10, IL1 $\beta$, IL- 18, ERa and SIRT1 antibody) and incubated overnight at $4^{\circ} \mathrm{C}$. An ECL chemiluminescence system was used to visualize signals using a computerized image processing software (Sunnyvale, CA).

\section{Statistical}

Assays were repeated three to five times and represented as mean + standard deviation (S. D.). ANOVA (analysis of variance) was used to examine significant differences of varied groups. A P-value below 0.05 was considered statistically significant.

\section{Results}

\section{Estrogen supplement attenuates MCAO-induced cerebral neuron injury in rats}

The MCAO group attained a mean score of $3.40 \pm 0.55$ with mean infarct volume of $22.46 \pm 1.90 \mathrm{~mm}^{3}$ thus both scores confirming positive formation by MCAO of cerebral ischemia-reperfusion damage. The M\&OVA group attained a mean score of $3.60 \pm 0.55$ with mean infarct volume of $25.33 \pm 1.81 \mathrm{~mm}^{3}$ with a score of $1.40 \pm 0.89$ for the estrogen treatment group with a mean infarct volume of $7.98 \pm 0.53 \mathrm{~mm}^{3}$ when compared to the MCAO group (Figures $1 \mathrm{~A}$ and $1 \mathrm{C}$ ). In support of these results, LDH production measured by the LDH assay was noted in the MCAO group ischemic hemisphere but was higher in the M\&OVA group and decreased in the estrogen group (Figure 1B). Moreover, the increased number of neurons attaining a normal morphology in the estrogen treatment group coincided with greater numbers of neurons dying in the M\&OVA group compared to those in the MCAO group (Figure 1D). Overall, these results indicated that the reduction in estrogen levels following ovariectomization can exacerbate cerebral ischemia-reperfusion damage and that this damage would be improved on by estrogen supplements.

\section{Estrogen supplement inhibits NLRP3 inflammasome activation during cerebral ischemia-reperfusion injury}


Western blot analysis employed for the examination of inflammation-associated cytokines expression per group after MCAO in rats to determine ERa's role in neuroinflammation. Significant reduction in ERa expression noted following MCAO as illustrated in Figure 2A and 2B. When compared against the MCAO group, the M\&OVA group demonstrated highly reduced levels of ERa protein, whereas, these levels reached high levels following estrogen supplementation (Figures $2 \mathrm{~A}$ and $2 \mathrm{~B}$ ). Moreover, MCAO and M\&OVA treatment led to high increases in NLRP3 inflammasome production (including ASC, NLRP3, IL$1 \beta$ and IL-18) and activation of caspase -1 . This was particularly true following ovariectomy. Moreover, when estrogen supplementation was given after MCAO treatment in rats, NLRP3 inflammasome and caspase-1 p10 levels were inhibited. Simultaneously, apoptotic cell percentages were observed to be comparable to expression levels of NLRP3 (Figure 2E and 2F). These findings demonstrate the crucial part for ERa in MCAO damage-induced neuroinflammation.

\section{ERa rescuing hydrogen peroxide-induced neuronopathies via SIRT1-dependent in vitro}

PC12 cell transfection with ERa plasmid was performed to examine potential processes occurring due to ERa activation and neuron damage. Neuronopathies were initiated by hydrogen-peroxide treatment after 24hrs and ERa levels examined by Western blot analysis. This indicated efficacious elevation of ERa levels following transfection of cultured PC12 cells (Figures $3 \mathrm{~A}$ and $3 \mathrm{~B}$ ). Moreover, additional findings of the FCM assay (Figures $3 \mathrm{C}$ and $3 \mathrm{~d}$ ) and the LDH assay (Figure $3 \mathrm{E}$ ) on PC12 cells demonstrated that when compared to the hydrogen-peroxide treatment group, hydrogen peroxide-instigated cell death and LDH production were prevented by ERa overexpression. Thus, indicating ERa neuroprotective impact on neuronal damage in vitro.

Previous studies performed in this laboratory demonstrated significant SIRT1 level reductions following MCAO. When contrasted against MCAO, the M\&OVA group showed greatly decreased SIRT1 protein expression levels which were efficaciously elevated with estrogen supplementation (Figure 3A and 3B). This demonstrated the close association between estrogen anti-neuronopathies and SIRT1. Furthermore, co-transfection of ERa with either SIRT1 or SIRT1 siRNA was performed to demonstrate the involvement of the SIRT1-dependent pathway in vivo in ERa's protection against neuronopathies. Figure 3 illustrates the outcome indicating that SIRT1 overexpression with ERa in a hydrogen-peroxide environment, resulted in decreases in neuron apoptosis and LDH production continuously and this was eradicated via SIRT1 siRNA and ERa co-transfection. Thus, these findings demonstrate neuronal activity regulation by ERa via the SIRT1-dependent pathway and as a response to hydrogen peroxide treatment.

\section{SIRT1 cooperates with ERa to counteract NLRP3 inflammasome activation}


ERa overexpression was achieved by transfection of cells with an ERa wildtype plasmid which was performed to examine ERa response to NLPR3-dependent neuroinflammation and caspase- 1 activation. PC12 cell's expression of cleaved caspase- 1 and NLRP3 inflammasome was highly decreased following ERa treatment. Next, PC12 cells SIRT1 levels were raised by transfection using wildtype SIRT1 plasmid in the presence of ERa overexpression. This was performed to establish whether maintenance of neuroinflammatory-associated gene on anti-nerve damage mediated by hydrogen peroxide requires SIRT1. From the caspase- 1 activity, it was evident that downregulation of NLRP3 inflammasome expression was attained in PC12 cells compared to those transfected as described. Contrastingly, SIRT1 siRNA transfection resulted in SIRT1 knockdown in the presence of ERa overexpression, and consequently, this resulted in higher inflammasome expression. Overall, these findings indicate the crucial part for SIRT1 in ERa's impact on the anti-neuroinflammatory factor expression.

\section{ERa-evoked anti-neuronopathies though SRIT1 is mediated by AMPK}

Earlier research indicated regulation of AMPK activation by estrogen in vivo ${ }^{40]}$. Compound $\mathrm{C}$ was employed to obstruct the AMPK pathway to allow evaluation of whether an association existed between the AMPK pathway and ERa's anti-neuro damage impact. Examining PC12 cells exposed to hydrogenperoxide found that ERa decreased neuronal damage levels. This was further backed by elevated cell viability levels and lower apoptotic-positive cell percentages and LDH production. Thus, compound $\mathrm{C}$ was found to suppress ERa' neuroprotective properties (Figure 5A and 5B), thereby indicating the principal role for AMPK in neuron survival due to ERa. Earlier studies in this lab indicated the need for SIRT1 in ERamediated neuroprotection. Consequently, this led to the hypothesis that ERa's neuroprotection was facilitated by the SIRT1-AMPK pathway with AMPK forming a strong relationship between ERa and SIRT1. Moreover, increased AMPK activation resulted from SIRT1 overexpression and AMPK phosphorylation mediated by ERa. Conversely, neurons where SIRT1 was suppressed demonstrated reduced AMPK expression thereby demonstrating that in hydrogen peroxide-treated neurons AMPK activation was damaged in conditions of SIRT1 absence.

\section{Discussion}

This study demonstrated the effect of ERa treatment and transfection using ERa plasmid in conferring neuroprotectivity against hypoxia/ischemic damage in vitro and in vivo. Findings indicated great improvements in neurological scores and lower infarct volumes following higher ERa and SIRT1 expression levels resulting from estrogen treatment. Moreover, estrogen treatment was found to inhibit NLRP3 inflammasome activation resulting in reduced cerebral ischemic/reperfusion-instigated neuronal damage. Contrastingly, ERa and SIRT1 deficit caused by ovariectomization led to direct elevation in neurological shortfalls, lessened in vivo infarct volume and initiated NLRP3 pathway factors expression indicating the involvement of ERa and SIRT1 activation in post-brain damage neuroinflammation. Outcomes of this study also indicated how abolishing SIRT1 ended the impact of ERa in inflammasomes, 
thus pointing to the SIRT1-dependent pathway of ERa's neuroprotective properties. Overall, this indicates that less hypoxia/ischemic damage would ensue through the action of ERavia the SIRT1-dependent pathway and by inhibition of NLRP3 inflammasome activation.

The ERa and ER $\beta$ receptors through which estrogen manifests its actions are part of the nuclear receptor (NR) superfamily [27]. The binding of estrogen to ER led to the ER's interaction with a particular DNA response element leading to the transcription of specific genes ${ }^{[28]}$. Notwithstanding the significance of both ERa and ERß actions, various studies have indicated contrasting outcomes in relation to their role in ischemic protection, with increasing levels of evidence pointing towards copious ERa at the RNA and protein levels ${ }^{[29-31]}$ and its central status in neuronal survival. One study indicated that lower ERa, not

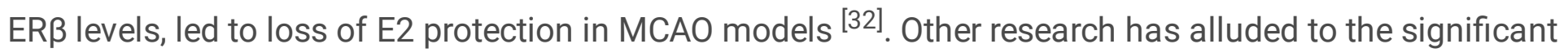
role of ERa rather than ERß in ischemic protection ${ }^{[33]}$ and that certain ERa, not ER $\beta$ was also neuroprotective ${ }^{[34]}$. These studies outline the greater part ERa holds concerning cerebral ischemic/reperfusion damage compared to ERß.

In support of past research, the findings of this study demonstrated that ERa shortage following ovariectomization led to in vivo decrease in infarct volume and elevated neurological insufficiency. Contrastingly, estrogen treatment resulted in enhancement of ERa leading to smaller cerebral infarct volume and better neurobehavioral results following stimulation by cerebral ischemia/reperfusion. Thus, this demonstrates the direct association between ERa activation and cerebral ischemia/reperfusion damage. Protection against hydrogen peroxide-induced nerve damage facilitated through ERa overexpression protection was examined to determine if ERa is a primary element conferring neuroprotection for neuronal survival using PC12 cells. Findings indicated efficacious enhancement of cell viability and suppressed LDH production in vitro by ERa, thereby demonstrating the protective impacts of ERa against hydrogen peroxide-induced neuronal injury.

Neuroinflammation is a byproduct of cerebral ischemia-reperfusion that provokes neuronal death together with elevated levels of IL-1 $\beta$ and IL-18 in the ischemic hemisphere ${ }^{[35,36]}$. Earlier research alluded to the progress of ischemia/reperfusion-induced brain damage because of the crucial part played by the NLRP3 inflammasome ${ }^{[11,12]}$. Ischemic stroke leads to elevation of NLRP3 inflammasome associated proteins' expression [37], whilst reducing NLRP3 inflammasome expression can decrease the likelihood of cerebral ischemia-induced neuronal injury ${ }^{[38]}$. As caspase- 1 activation and IL-1 $\beta$ production are impacted by the NLRP3 inflammasome, these inflammasomes were assessed for partaking in cerebral ischemic/reperfusion damage and if NLRP3 inflammasome activation is inhibited by ERa. The finding of this study indicated significant elevation in NLRP3 inflammasome expression in brains at times of cerebral ischemia/reperfusion, whilst ERa shortage following ovariectomization enhances caspase- 1 activation and generation. Contrastingly, significant decrease in NLRP3 inflammasome (e.g. NLRP3, caspase-1 p10, ASC in vivo and invitro) expression was brought about by transfection with ERa plasmid or estrogen treatment. Furthermore, higher levels of ERa decreased IL-18 
and IL-1 $\beta$ levels. Overall, these findings indicated an efficient increase in neuronal survival by ERa against neuroinflammation.

The crucial role played by SIRT1 in the inflammatory response is widely acknowledged with several studies suggesting SIRT1 overexpression highly suppressed NLRP3 inflammasome activation in vascular endothelial cells ${ }^{[18]}$ and dextran sodium sulfate-related rhapontin increased NLRP3 inflammasome protein expression by the SIRT1-dependent pathway ${ }^{[39]}$. Large research data also indicates a vital need for SIRT1 as a neuroprotective element combatting cerebral ischemic/reperfusion damage. This study's findings were in keeping with this demonstrating elevation of SIRT1 expression together with NLRP3 inflammasome suppression following estrogen treatment. Interestingly, the neuroprotective impact of ERa against damage can be eliminated by SIRT1 silencing. SIRT1 silencing was also implicated in annulling the lowering of NLRP3 inflammasome activation induced by ERa, thereby signifying a relationship between SIRT1 signaling and ERa protective impacts.

Neuronal cell energy levels are depleted as a result of brain ischemia and the presence of barriers to energy depletion enhances the outcome of brain damage resulting from ischemic stress ${ }^{[40]}$. AMPK functions to preserve energy and is, therefore, a master metabolic switch with one study signifying the crucial part played by the SIRT1-AMPK signaling pathway in resisting ischemic stroke ${ }^{[41]}$. Whilst a separate study demonstrated ERa's suppression of neuronal injury facilitated by ischemic stroke using the SIRT1-dependent AMPK pathway ${ }^{[42]}$. Findings of this study further strengthened this argument by demonstrating the increase of AMPK activation by ERa and the elimination of ERa's neuroprotective qualities following the action of AMPK inhibitors. Most significantly, the findings concerning PC12 cells established that AMPK and SIRT1 took part in ERa neuroprotection processes and that ERa-induced AMPK activation was reliant on SIRT1. Overall, these findings indicate the significance of the SIRT1AMPK signaling pathway in ERa's neuroprotection.

In summary, suppression of ischemic-induced NLRP3 inflammasome expression and protection against cerebral ischemic damage both resulted from ERa activation together with SIRT1-AMPK pathway activation. These findings contribute new insights into ERa processes involved in ischemic stroke damage, thereby indicating possible therapeutic targets for stroke inhibition in postmenopausal women.

\section{Conclusion}

The findings indicate that inhibition of the NLRP3 inflammasome by ERa results in neuroprotection against hypoxia/ischemic injury and that ERa's neuroprotection can be highly improved by the SIRT1dependent AMPK pathway.

\section{Abbreviations}

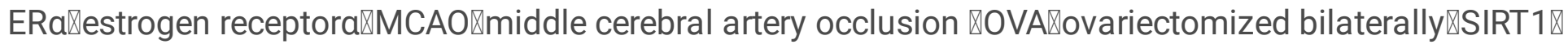

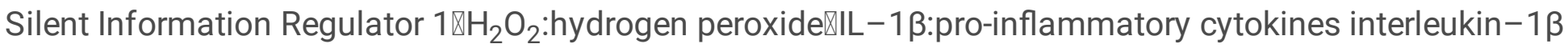




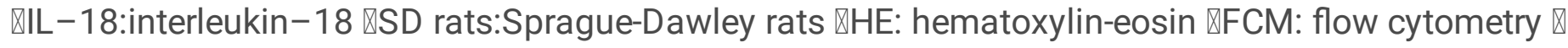
ANOVA:analysis of variance『NR: nuclear receptor

\section{Declarations}

\section{Ethics approval and consent to participate}

The study was approved by the Medical ethics committee of Wuhan university of science and technology for scientific research projects. \Audit section no.(201961) $\square$

Ethical review opinions as follows: By the hospital ethics committee review, the research of experimental design and scheme fully considering the safety and fairness principle, fully embodies the experiment by replacing, reduction and optimization of three principles, rights and interests to protect animals and will ease the pain of animals to the greatest extent, pain and stress, there is no conflict of interest between research content and research results.

\section{Consent for publication}

Not applicable.

\section{Availability of data and materials}

The datasets used and/or analysed during the current study are available from the corresponding author on reasonable request.

\section{Competing interests}

The authors declare that they have no competing interests.

\section{Funding}

Funding for this study was provided by the Research Program of Hubei Province Key Laboratory of Occupational Hazard Identification and Control (OHIC2018K01),

Funder囚Chen YJ. In this study, Chen YJ participated to the writing of the manuscript, data analysis and marking of the final version;

The Zhejiang Medical and Health Scientific Research Project(2018KY661),

Funder: Z Z. In this study, Z Z designed the study, participated to data analysis. 


\section{Authors' contributions}

Ze Zhong designed the study, participated to data analysis $₫$ Wenqi He participated to animals feeding and behavior test; Xiaolu Cao took part to build animal models and protein detection; Bei Tang participated to animals feeding and morphological detection; Qianya Fan took part to cell culture and flow cytometry test; Minghui Zhao took part to cell culture and plasmid transfection experiment; Yajun Chen participated to the writing of the manuscript, data analysis and marking of the final version.

\section{Acknowledgements}

Not applicable.

\section{Authors' information}

${ }^{1}$ Department of Cardiology, Jiande Branch, Second Affiliated Hospital, Zhejiang University School of Medicine, Jiande, Zhejiang 311600, China.

${ }^{2}$ Hubei Province Key Laboratory of Occupational Hazard Identification and Control, Department of pharmacology, Medical college of Wuhan University of Science and Technology, Wuhan, Hubei, 430080, China.

\#Z Z and He WQ contributed equally to this study and shared the first authorship.

*Correspondences to: Z Z凶Department of Cardiology, Jiande Branch, Second Affiliated Hospital, Zhejiang University School of Medicine, Jiande, Zhejiang 311600, China.zhongze1012@163.com

Chen YJ, Hubei Province Key Laboratory of Occupational Hazard Identification and Control, Department of pharmacology, Medical college of Wuhan University of Science and Technology, Wuhan, Hubei, 430080, China. Chenrunnerup@163.com

\section{References}

1. P. Langhorne, J. Bernhardt, G. Kwakkel. Stroke rehabilitation[J]. Lancet, 2011, 14, 377(9778): 1693702.

2. J. Chen, R. K. Leak, G. Y. Yang. Perspective for stroke and brain injury research: mechanisms and potential therapeutic targets[J]. CNS NeurosciTher, 2015, 21(4): 301-3.

3. D. Y. Fann, et al. Pathogenesis of acute stroke and the role of inflammasomes[J]. Ageing Res Rev, 2013, 12(4):941-66.

4. W. Chumboatong, et al. Neuroprotection of agomelatine against cerebral ischemia/ reperfusion injury through an antiapoptotic pathway in rat[J].Neurochem Int, 2017, 102:114-122. 
5. K. M. Buckley, et al. Rapamycin up-regulation of autophagy reduces infarct size and improves outcomes in both permanent MCAL, and embolic MCAO, murine models of stroke[J]. Exp Transl Stroke Med, 2014, 21, 6:8.

6. P. Zhuang, et al. Salvianolic Acids for Injection (SAFI) suppresses inflammatory responses in activated microglia to attenuate brain damage in focal cerebral ischemia[J]. J Ethnopharmacol, 2017, 23, 198:194-204.

7. G. Santoni, et al. Danger- and pathogen-associated molecular patterns recognition by patternrecognition receptors and ion channels of the transient receptor potential family triggers the inflammasome activation in immune cells and sensory neurons[J].J Neuroinflammation, 2015, 3, $12: 21$.

8. Ives A, Nomura J, Martinon F, Roger T, LeRoy D, Miner JN, Simon G, Busso N, So A. Xanthine oxidoreductase regulates macrophage IL1 secretionupon NLRP3 inflammasome activation[J]. Nat Commun, 2015, 24, 6: 6555.

9. Song L, Pei L, Yao S1, Wu Y, Shang Y. NLRP3 Inflammasome in Neurological Diseases, from Functions to Therapie[J]. Front Cell Neurosci, 2017, 9,11: 63.

10. Qiu Z, Lei S, Zhao B, Wu Y, Su W, Liu M, Meng Q, Zhou B, Leng Y, Xia ZY. NLRP3 Inflammasome Activation-Mediated Pyroptosis Aggravates Myocardial Ischemia/Reperfusion Injury in Diabetic Rats[J]. Oxid Med Cell Longev, 2017, 2017: 9743280.

11. J. Qiu, et al. The neuroprotection of Sinomenine against ischemic stroke in mice by suppressing NLRP3 inflammasome via AMPK signaling[J]. Int Immunopharmacol, 2016, 40:492-500.

12. X. Wang, et al. Umbelliferone ameliorates cerebral ischemia-reperfusion injury via upregulating the PPAR gamma expression and suppressing TXNIP/NLRP3 inflammasome[J].Neurosci. Lett, 600 (2015) 182-187.

13. M. C. Haigis, D. A. Sinclair. Mammalian sirtuins: biological insights and disease relevance[J]. Annu Rev Pathol, 2010, 5:253-95.

14. M. Hernandez-Jimenez, O. Hurtado, M. I. Cuartero, I. Ballesteros, A. Moraga, J. M. Pradillo, M. W. McBurney, I. Lizasoain, M. A. Moro. Silent information regulator 1 protects the brain against cerebral ischemic damage[J]. Stroke, 2013, 44(8):2333-7.

15. H. Lv, et al. Salvianolic acid B attenuates apoptosis and inflammation via SIRT1 activation in experimental stroke rats[J].Brain Res Bull, 2015, 115:30-6.

16. Y. Yang, et al. Melatonin prevents cell death and mitochondrial dysfunction via a SIRT1-dependent mechanism during ischemic-stroke in mice[J]. J Pineal Res, 2015, 58(1):61-70.

17. C. H. Ma, et al. Simiao pill ameliorates renal glomerular injury via increasing Sirt1 expression and suppressing NF-kappaB/NLRP3 inflammasome activation in high fructose-fed rats[J]. J Ethnopharmacol, 2015, 22, 172:108-17.

18. Y. Li, et al. SIRT1 inhibits inflammatory response partly through regulation of NLRP3 inflammasome in vascular endothelial cells[J]. Mol Immunol, 2016, 77:148-56. 
19. Nilsson S, Mäkelä S, Treuter E, Tujague M, Thomsen J, Andersson G, Enmark E, Pettersson K, Warner M, Gustafsson JA. Mechanisms of estrogen action[J]. Physiol Rev, 2001, 81(4):1535-65.

20. Wang L, Andersson S, Warner M, Gustafsson JA. Estrogen receptor (ER)beta knockout mice reveal a role for ERbeta in migration of cortical neurons in the developing brain[J]. Proc. Natl. Acad. Sci. U.S. A, 2003, 100: 703-708.

21. Wise PM, Dubal DB, Wilson ME, Rau SW, Bottner M, Rosewell KL. Estradiol is a protective factor in the adult and aging brain: understanding of mechanisms derived from in vivo and in vitro studies[J]. Brain Res. Rev, 2001, 37: 313-319.

22. Dubal DB, Rau SW, Shughrue PJ, Zhu H, Yu J, Cashion AB, Suzuki S, Gerhold LM, Bottner MB, Dubal SB, Merchanthaler I, Kindy MS, Wise PM. Differential modulation of estrogen receptors (ERs) in ischemic brain injury: a role for ERalpha in estradiol-mediated protection against delayed cell death[J]. Endocrinology, 2006, 147: 3076-3084.

23. Dietrich AK, Humphreys GI, Nardulli AM. Expression of Estrogen Receptor a in the Mouse Cerebral Cortex[J]. Mol Cell Endocrinol, 2015,5, 406: 19-26.

24. Thakkar R, Wang R, Sareddy G, Wang J, Thiruvaiyaru D, Vadlamudi R, Zhang Q, Brann D. NLRP3 Inflammasome Activation in the Brain after Global Cerebral Ischemia and Regulation by $17 \beta$ Estradiol[J]. Oxid Med Cell Longev, 2016, 2016: 8309031.

25. Stokes WS. Humane endpoints for laboratory animals used in regulatory testing[J]. ILAR J,2002,43:S31-8

26. Canadian Council on Animal Care (CCAC). CCAC guidelines on: euthanasia of animals used in science[C]. Ottawa: K1P 5 G4.

27. Wang C, Jie C, Dai X. Possible roles of astrocytes in estrogen neuroprotection during cerebral ischemia[J]. Rev Neurosci, 2016;25:255-268.

28. M.-A. Arevalo, I. Azcoitia, and L. M. Garcia-Segura. The neuroprotective actions of oestradiol and oestrogen receptors[J]. Nature Reviews Neuroscience, 2015, 16(1): 17-29.

29. Naugle MM, Nguyen LT, Merceron TK, Filardo E, Janssen WG, Morrison JH, Rapp PR, Gore AC. Gprotein coupled estrogen receptor, estrogen receptor $a$, and progesterone receptor immunohistochemistry in the hypothalamus of aging female rhesus macaques given long-term estradiol treatment[J]. J Exp ZoolAEcol Genet Physiol, 2014, 321(7): 399-414.

30. Westberry JM, Wilson ME. Regulation of estrogen receptor alpha gene expression in the mouse prefrontal cortex during early postnatal development[J]. Neurogenetics, 2012, 13(2): 159-67.

31. Thakur MK, Sharma PK. Transcription of estrogen receptor alpha and beta in mouse cerebral cortex: effect of age, sex, 17beta-estradiol and testosterone[J]. Neurochem Int, 2007, 50(2): 314-21.

32. Dubal DB, Zhu H, Yu J, Rau SW, Shughrue PJ, Merchenthaler I, Kindy MS, Wise PM. Estrogen receptor alpha, not beta, is a critical link in estradiol-mediated protection against brain injury[J]. Proc Natl Acad Sci U S A, 2001, 13, 98(4):1952-7.

33. Zhang QG, Raz L, Wang R, Han D, De Sevilla L, Yang F, Vadlamudi RK, Brann DW. Estrogen attenuates ischemic oxidative damage via an estrogen receptor alpha-mediated inhibition of NADPH oxidase 
activation[J]. J Neurosci, 2009, 4, 29(44):13823-36.

34. Dai X, Chen L, Sokabe M.Neurosteroid estradiol rescues ischemia-induced deficit in the long-term potentiation of rat hippocampal CA1 neurons[J]. Neuropharmacology, 2007,52(4):1124-38.

35. H. Shan, Y. Bian, Z. Shu, L. Zhang, J. Zhu, J. Ding, M. Lu, M. Xiao, G. Hu. Fluoxetine protects against IL-1beta-induced neuronal apoptosis via downregulation of p53[J]. Neuropharmacology, 2016, 107:68-78.

36. M. J. Zhang, Y. Zhou, X. Wang, X. Chen, Y. Pi, L. Guo, C. Y. Gao, J. C. Li, L. L. Zhang. Interleukin-18 gene promoter $607 \mathrm{~A}$ polymorphism, but not $137 \mathrm{C}$ polymorphism, is a protective factor for ischemic stroke in the Chinese population: a meta-analysis[J].Meta Gene, 2016, 29, 9:165-72.

37. K. Zhou, L. Shi, Y. Wang, S. Chen. Recent Advances of the NLRP3 Inflammasome in Central Nervous System Disorders[J]. J Immunol Res, 2016, 2016:9238290.

38. F. Yang, Z. Wang, X. Wei, H. Han, X. Meng, Y. Zhang, W. Shi, F. Li, T. Xin, Q. Pang, F. Yi. NLRP3 deficiency ameliorates neurovascular damage in experimental ischemic stroke[J]. J Cereb Blood Flow Metab, 2014, 34(4):660-7.

39. W. Wei, et al. Rhapontin ameliorates colonic epithelial dysfunction in experimental colitis through SIRT1 signaling[J]. Int Immunopharmacol, 2017, 42:185-194.

40. Taxin ZH, Neymotin SA, Mohan A, Lipton P, Lytton WW. Modeling molecular pathways of neuronal ischemia[J]. Prog Mol Biol Transl Sci, 2014, 123:249-275.

41. P. Wang, et al. Nicotinamide phosphoribosyl transferase protects against ischemic stroke through SIRT1-dependent adenosine monophosphate-activated kinase pathway[J]. Ann Neurol, 2011, 69(2):360-74.

42. Guo J-M, Shu H, Wang L, Xu J-J, Niu X-C, Zhang L. SIRT1-dependent AMPK pathway in the protection of estrogen against ischemic brain injury[J]. CNS NeurosciTher, 2017, 23:360-369.

\section{Figures}


A

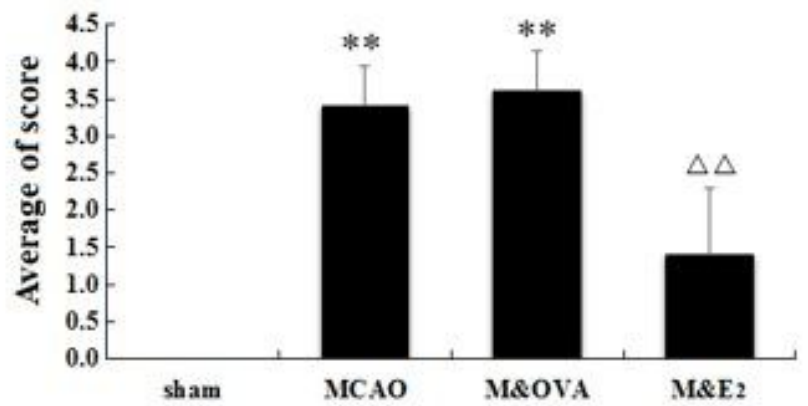

C

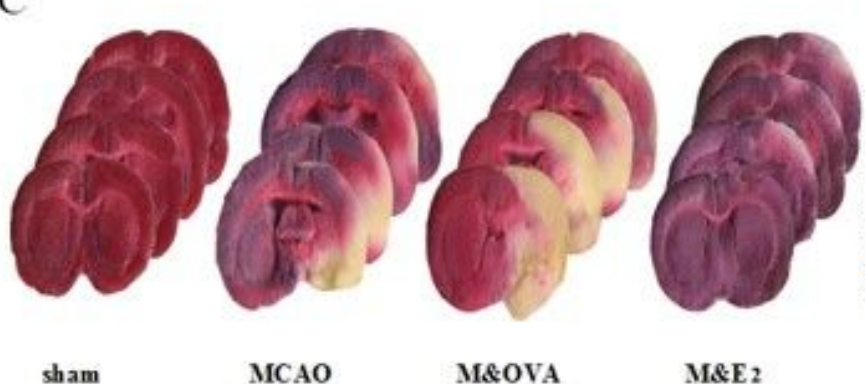

$\mathrm{D}$

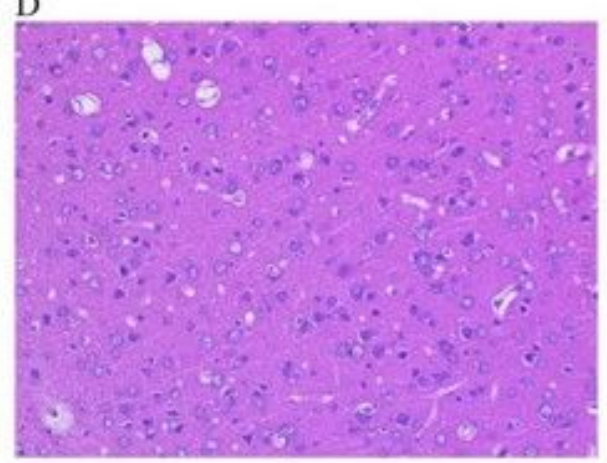

sham

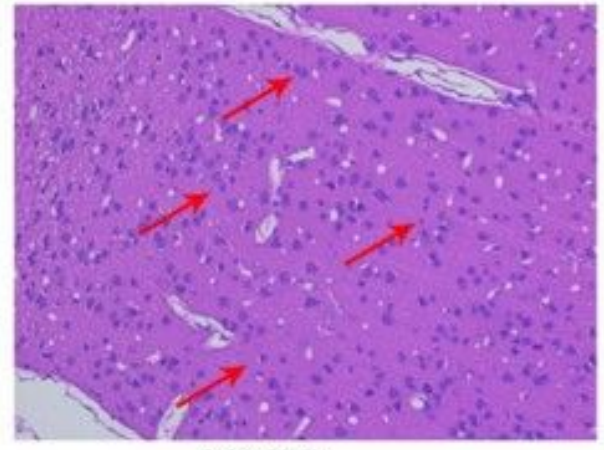

M\&OVA
B
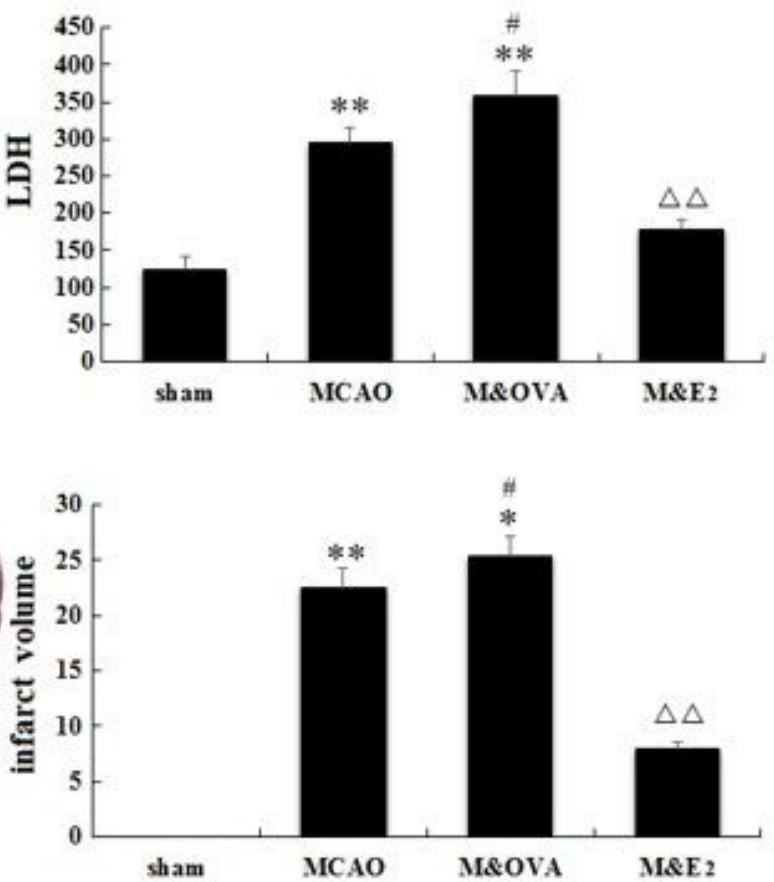

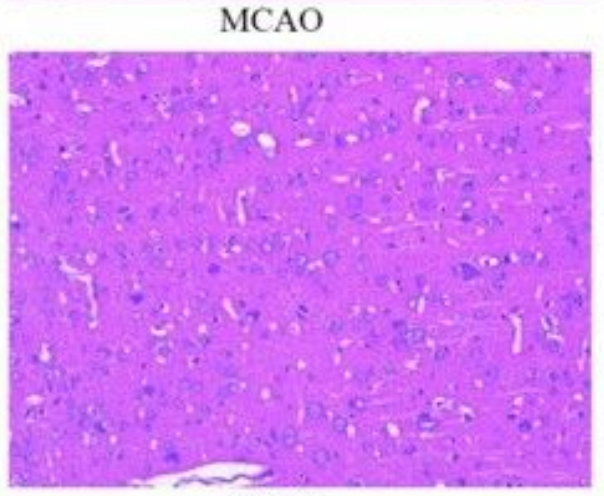

$M \& E_{2}$

\section{Figure 1}

Illustration of Longa's behavioral analyses (A), LDH assay (B), TTC stained infarct volume assessment (C) and HE staining (D) per group. M\&E2:estrogen supplement prior to MCAO, M\&OVA: ovariectomized prior to MCAO. Dead neurons indicated by red arrows. Data are given as mean \pm SD for triplicate assays, $n=5,{ }^{*} P<0.05,{ }^{\star} \times P<0.01$ vs sham group, $\# P<0.05$ vs $M C A O$ group, $\triangle \triangle P<0.01$ vs $M \& 0 V A$ group. 


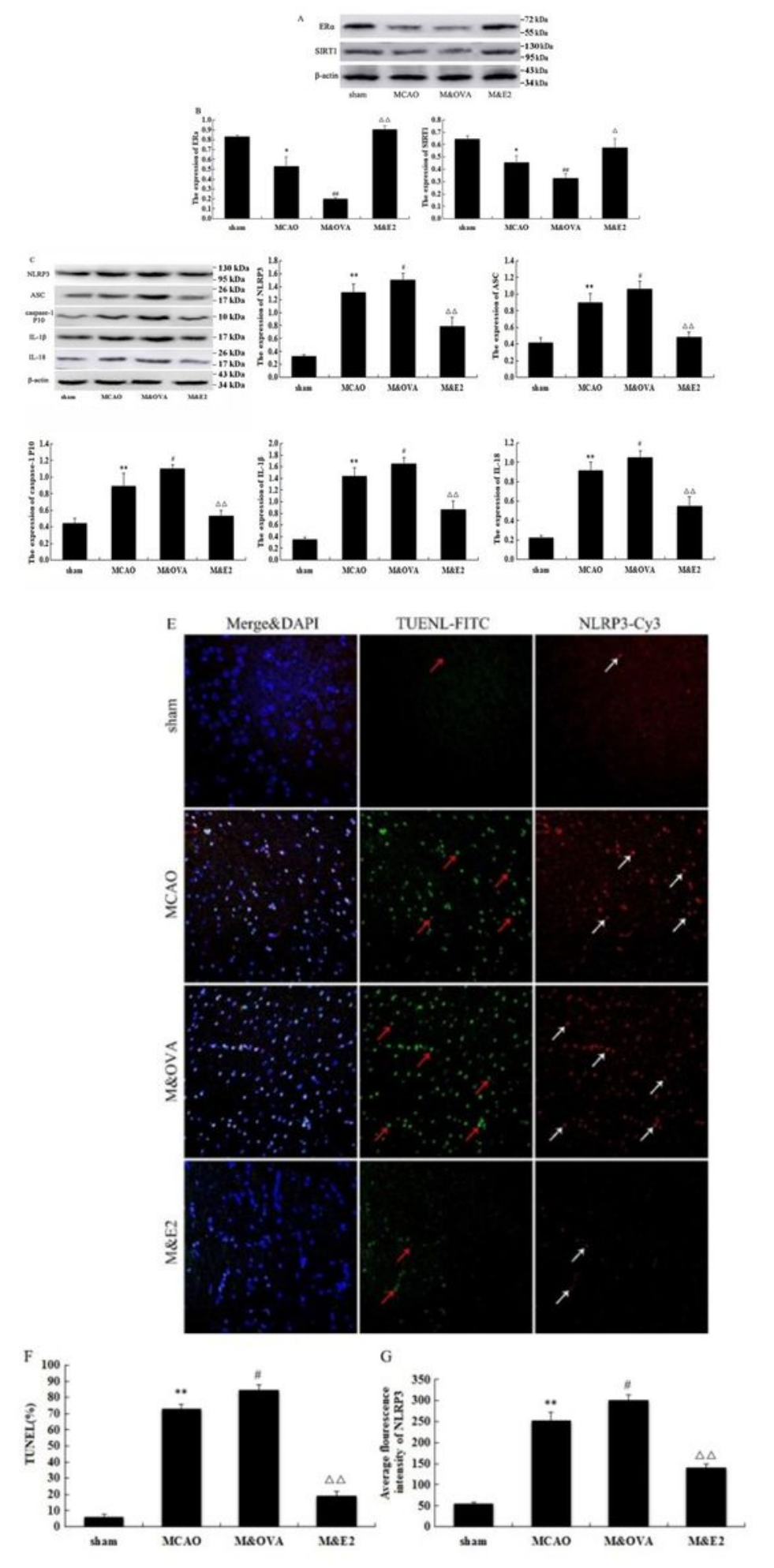

Figure 2

Western blot analysis of expression of ERa (A and B) and NLRP3, ASC, caspase-1 P10, IL-1 3 and IL-18 (C and $D$ ). Protein expression levels are given arbitrarily and results normalized to the related $\beta$-actin protein quantity. Immunofluorescent image of TUNEL stained apoptotic cell percentage (green) (E and F) and expression of NLRP3 (red) (E and G). Positive apoptotic cells shown by red arrows, whilst white arrows are indicative of NLRP3 protein expression as observed in the same field. M\&E2:estrogen supplement 
before MCAO, M\&OVA: ovariectomized before MCAO. Data are given as mean \pm SD for triplicate assays, $n=5,{ }^{*} P<0.05,{ }^{\star \star} P<0.01$ vs sham group, $\# \# P<0.05$, $\# P<0.05$ vs $M C A O$ group, $\triangle \triangle P<0.01$ vs $M \& O V A$ group.
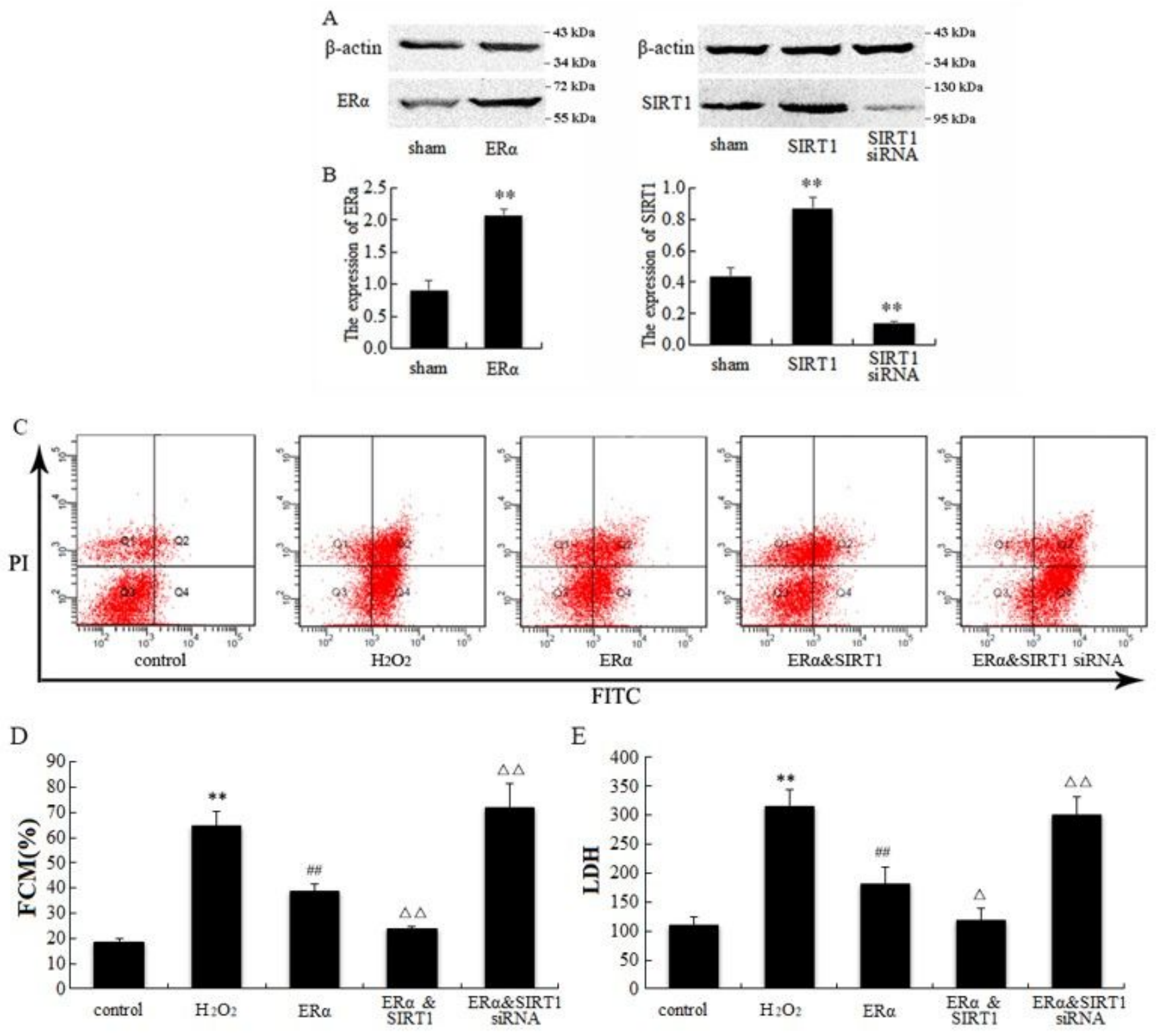

\section{Figure 3}

PC12 cells Western blot analysis of ERa and SIRT1 expression (A and B) and FCM assay examination of PC12 apoptotic cells ( $C$ and $D$ ). LDH production demonstrates LDH levels of culture media. The findings are of three individual assays and are mutually supportive. ERa: transfected with a wild-type Estrogen Receptor a $(0.5 \mu \mathrm{g})$ before $\mathrm{H} 2 \mathrm{O} 2$ treatment $(400 \mu \mathrm{M}$ for $12 \mathrm{~h})$, ERa\&SIRT1: co-transfected a wild-type SIRT1 $(0.5 \mu \mathrm{g})$ with Estrogen Receptor a $(0.5 \mu \mathrm{g})$ before $\mathrm{H} 2 \mathrm{O} 2$ treatment, ERa\&SIRT1 siRNA: co-transfected the SIRT1 small interference RNA $(0.5 \mu \mathrm{g})$ with Estrogen Receptor a $(0.5 \mu \mathrm{g})$ before $\mathrm{H} 2 \mathrm{O} 2$ treatment, $\mathrm{H} 2 \mathrm{O} 2$ : hydrogen peroxide. ${ }^{*} P<0.05$ vs sham group, \#\# $P<0.01$ vs $H 202$ group, $\triangle P<0.05, \triangle \triangle P<0.01$ vs $E R a$ group. 

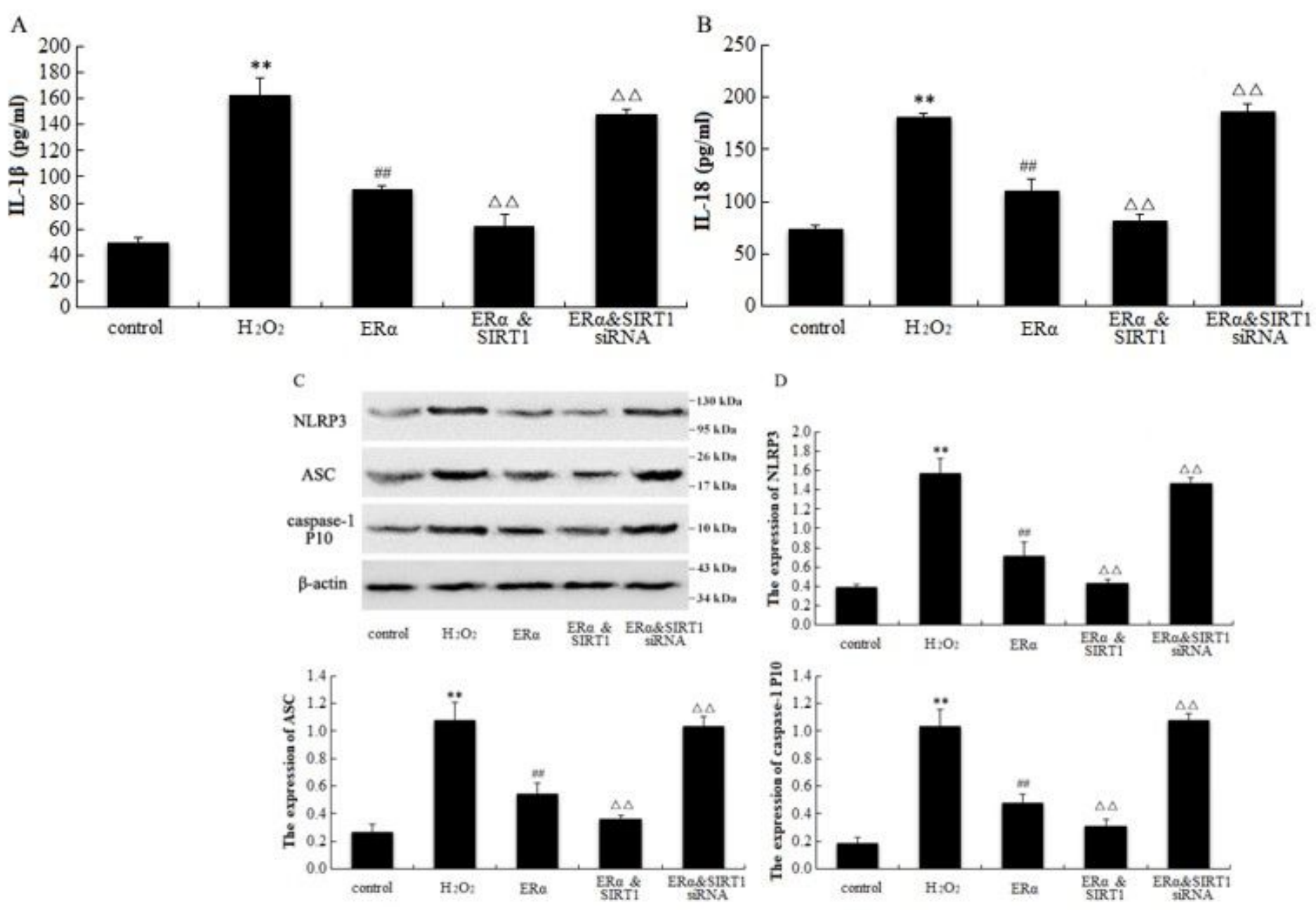

\section{Figure 4}

Quantification of the inflammatory cytokines IL-1 $\beta$ and IL-18 using ELISA ( $A$ and B). Western blot analysis of ASC, NLRP3, caspase-1 p10 expression in PC12 cells ( $C$ and D). The findings are of three individual assays and are mutually supportive. ERa: transfected with a wild-type Estrogen Receptor a $(0.5 \mu \mathrm{g})$ before $\mathrm{H} 2 \mathrm{O} 2$ treatment $(400 \mu \mathrm{M}$ for $12 \mathrm{~h})$, ERa\&SIRT1: co-transfected a wild-type SIRT1 $(0.5 \mu \mathrm{g})$ with Estrogen Receptor a $(0.5 \mu \mathrm{g})$ before $\mathrm{H} 2 \mathrm{O} 2$ treatment, ERa\&SIRT1 siRNA: co-transfected the SIRT1 small interference RNA $(0.5 \mu \mathrm{g})$ with Estrogen Receptor a $(0.5 \mu \mathrm{g})$ before $\mathrm{H} 2 \mathrm{O} 2$ treatment, $\mathrm{H} 2 \mathrm{O} 2$ : hydrogen peroxide. ${ }^{*} \mathrm{P}<0.01$ vs sham group, \#\# $\mathrm{P}<0.01$ vs $\mathrm{H} 2 \mathrm{O} 2$ group, $\triangle \triangle \mathrm{P}<0.01$ vs ERa group. 

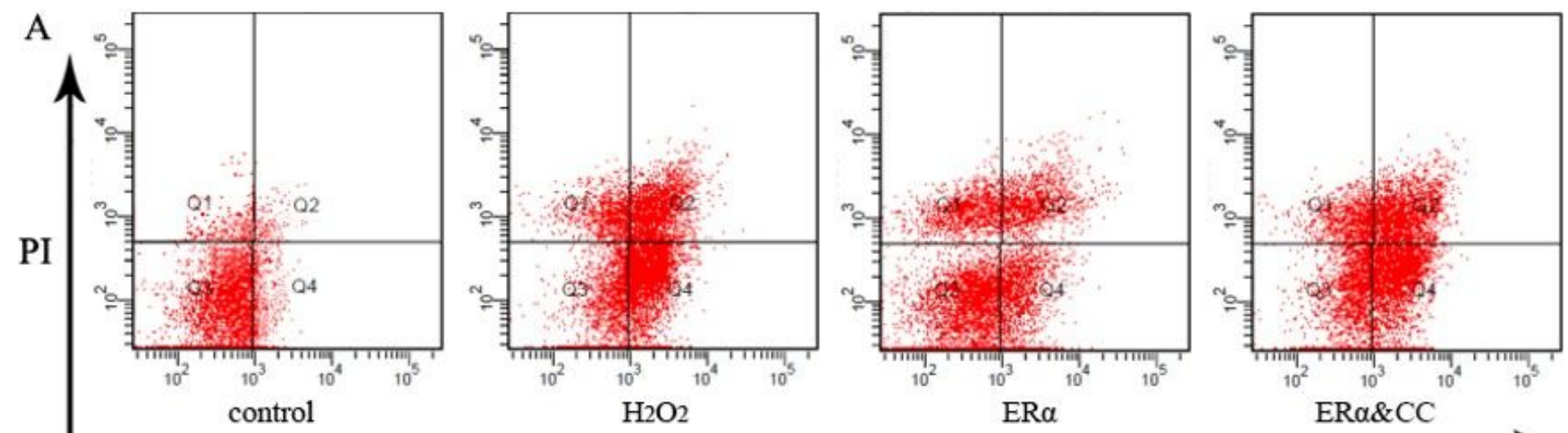

$\mathrm{ER} \alpha$

$\mathrm{ER} \alpha \& C \mathrm{C}$

\section{FITC}

B

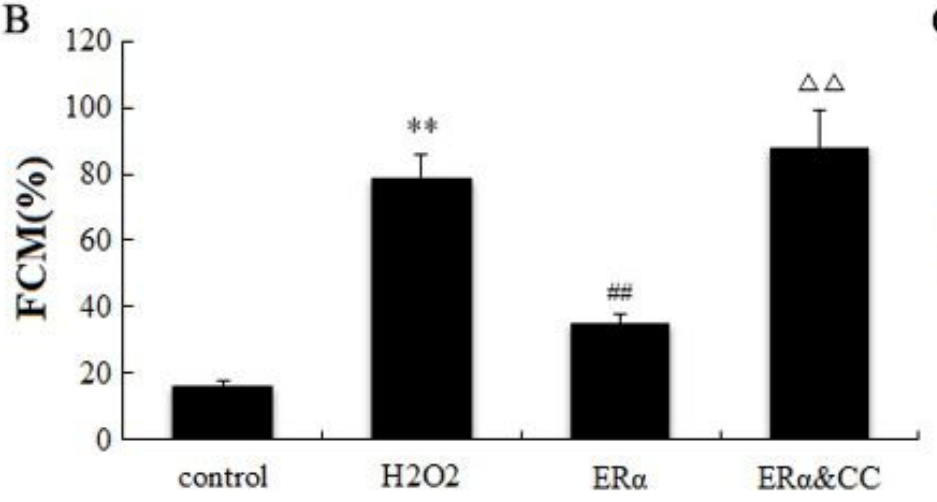

D

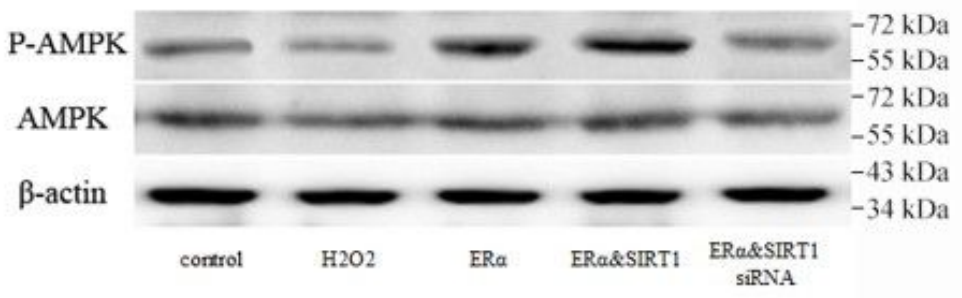

$\mathrm{C}$

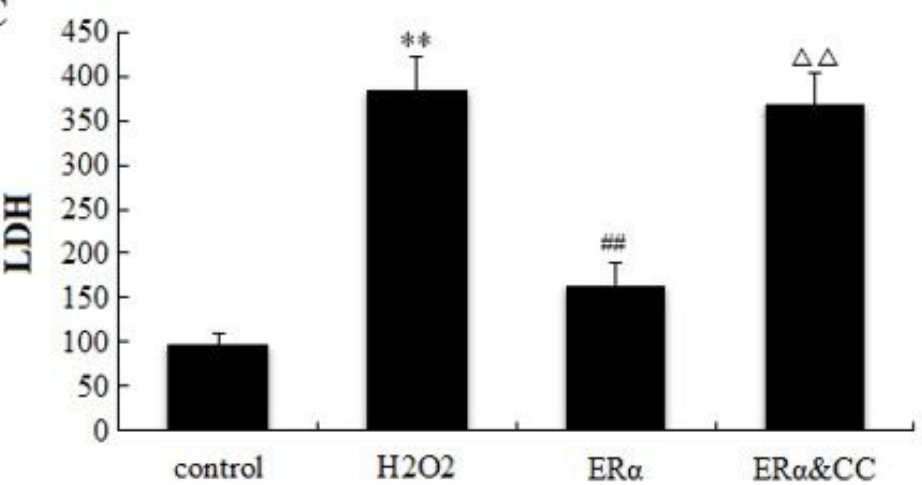

E

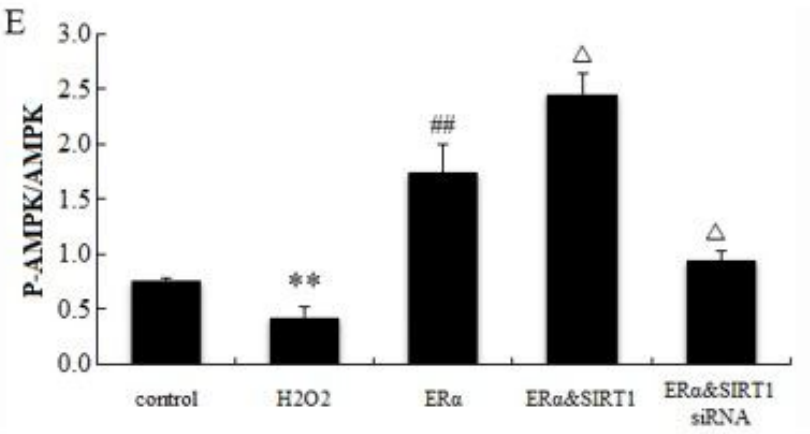

Figure 5

FCM assay utilised for observation of apoptotic PC12 cells (A and B), LDH production demonstrating LDH levels in culture media (C), Western blot analysis of PC12 cells for AMPK/P-AMPK expression levels ( $D$ and $E$ ). The findings are of three individual assays and are mutually supportive. ERa: transfected with a wild-type Estrogen Receptor a $(0.5 \mu \mathrm{g})$ before $\mathrm{H} 2 \mathrm{O} 2$ treatment $(400 \mu \mathrm{M}$ for $12 \mathrm{~h})$, ERa\&CC: AMPK-inhibitor Compound $\mathrm{C}$ treatment and transfected with a wild-type Estrogen Receptor a $(0.5 \mu \mathrm{g})$ before $\mathrm{H} 2 \mathrm{O} 2$ treatment, ERa\&SIRT1: co-transfected a wild-type SIRT1 $(0.5 \mu \mathrm{g})$ with Estrogen Receptor a $(0.5 \mu \mathrm{g})$ before $\mathrm{H} 2 \mathrm{O} 2$ treatment, ERa\&SIRT1 siRNA: co-transfected the SIRT1 small interference RNA $(0.5 \mu \mathrm{g})$ with Estrogen Receptor a $(0.5 \mu \mathrm{g})$ before $\mathrm{H} 2 \mathrm{O} 2$ treatment, $\mathrm{H} 2 \mathrm{O} 2$ : hydrogen peroxide. ${ }^{*} \mathrm{P}<0.05$ vs sham group, \#\# $\mathrm{P}<0.01$ vs $\mathrm{H} 2 \mathrm{O} 2$ group, $\triangle \mathrm{P}<0.05, \triangle \triangle \mathrm{P}<0.01$ vs ERa group.

\section{Supplementary Files}


This is a list of supplementary files associated with this preprint. Click to download.

- ARRIVEchecklist.xlsx 